\title{
Responsabilidad social de las empresas agroindustriales del sector arrocero en Villavicencio Colombia
}

\author{
Obando-Bastidas, Jorge Alejandro ${ }^{1}$ \\ Franco-Montenegro, Aldemar ${ }^{2}$ \\ Bohórquez-Barbosa, Tania Margarita ${ }^{3}$ \\ Figueroa-Enciso, Stefany Michel ${ }^{4}$
}

\section{Resumen}

La responsabilidad social empresarial, en las diversas organizaciones evidencia la protección de los derechos de la naturaleza y los seres humanos, de esta depende el beneficio colectivo. El objetivo propone evaluar la responsabilidad social asumida por las empresas arroceras del sector agroindustrial en Villavicencio, en los factores relacionados con la contaminación ambiental, la salud, la educación y vivienda para los diferentes actores comprometidos en este contexto laboral. Para cumplir con dicho propósito se aplicaron 700 encuestas estructurada tipo Likert a los habitantes de la comunidad aledaña y a los trabajadores de estas empresas. Utilizando el método estadístico multivariante del análisis de correspondencia se observó, bajo compromiso social en todos los aspectos en la comunidad y un leve compromiso para los trabajadores. El medio ambiente es uno de los aspectos de menor apoyo, evidenciado desde la comunidad y desde los trabajadores.

Palabras Clave: responsabilidad social empresarial; sector arrocero; trabajadores de la industria arrocera; comunidad aledaña a las industrias arroceras.

Recibido: 15.05.17 Aceptado:20.02.18

1 Magister en Estadística Aplicada (Universidad de Granada, España), Magister en educación. (Universidad central de Chile), Matemático. Ingeniero de sistemas, Docente universidad cooperativa de Colombia Facultad de Contradiría Publica. E-mail: jorge.obandob@campusucc.edu.co , Código Orcid: orcid.org/0000-0002-42832871

2 Docente Universidad Cooperativa de Colombia. Facultad de Ciencias Económicas, Administrativas y Contables. Economista, Contador Público, especializado en Revisoría Fiscal y Control de Gestión. Correo. Aldemar.Franco@campusucc.edu.co

3 Contador público en proceso de graduación, Universidad Cooperativa de Colombia (Villavicencio, Meta, Colombia). E-mail: tania.bohorqueszb@campusucc.edu.co

4 Contador público en proceso de graduación, Universidad Cooperativa de Colombia (Villavicencio, Meta, Colombia). E-mail: stefany.figueroa@campusucc.edu.co 


\title{
Social responsibility of agro-industrial companies in the rice sector in Villavicencio Colombia
}

\begin{abstract}
The Social Entrepreneurship (SE) in the different organizations evidences the human rights and nature protection, which depend on the collective benefit. The aim proposes to evaluate the social responsibility assumed by the rice companies of the agroindustrial sector in Villavicencio, in the factors related to environmental pollution, health, education and housing for the different actors involved in this labor context. These elements are assumed by the agricultural industries sector in Villavicencio, especially by the rice companies. In order to accomplish the aim of this project, 700 structured Likerttype surveys were applied to the surrounding community and to the workers of these companies. As well, by using the correspondence analysis multivariate statistical method was possible to observe low social engagement in all aspects, and a slight engagement for the workers. Indicated from the community and from the workers, the environment is one of the main aspects with lower support.
\end{abstract}

Keywords: Social responsibility business rice sector, workers in the rice industry, surrounding community rice industries

\section{Introducción}

El municipio de Villavicencio se encuentra geográficamente ubicado en el piedemonte de la Cordillera Oriental al Noroccidente del departamento del Meta (Colombia), cubre una superficie de $1.311 \mathrm{~km}^{2}$. La industria del arroz ha sido un factor que económicamente ha beneficiado en muchos aspectos a la comunidad de Villavicencio; sin embargo, alrededor de los 14 molinos de arroz activos, circulan arroyos de agua que terminan siendo contaminados por los diferentes químicos usados en el tratamiento de este producto. La cantidad de humo emanados por estos molinos y que salen por las chimeneas, generan desde contaminación ambiental hasta contaminación visual.
Por otro lado, los malos olores que se perciben alrededor de estos espacios se constituyen en evidencias de contaminación que provienen de las actividades desarrolladas por estas industrias. Hernández et al, (2016:43) manifiestan que, para estos problemas, se requiere de tecnología de punta para el procesamiento y secado de este producto que ayuden a contrarrestar los efectos nocivos de las plagas $y$ bacterias que atacan el arroz, las cuales terminan generando contaminación. De la misma manera es necesario que estas empresas contribuyan a enfrentar fenómenos climáticos como el del Niño, cuyo paso deja estrago y desolación (FEDEARROZ, 2010:9).

Desde esta perspectiva se pretende evaluar la responsabilidad 
social (en adelante RSE) asumidas por las empresas arroceras del sector agroindustrial en Villavicencio, en los factores relacionados con la contaminación ambiental, la salud, la educación y vivienda para los diferentes actores comprometidos en este contexto laboral. Dicho compromiso nace directamente de los principios de la RSE, que deben ser cumplidos por las diferentes empresas dedicadas a la realización de estos procedimientos.

La investigación es de orden cuantitativo, se apoya en la estadística aplicando métodos de la correspondencia. Se estudian empresas del sector arrocero, considerando aspectos relativos a los ámbitos: educación, vivienda, medio ambiente, salud y recreación. Fue diseñada una encuesta, la cual se aplicó a 4 empresas del sector arrocero de la ciudad de Villavicencio, dos tipos de poblaciones: trabajadores.

\section{Responsabilidad Social Empresarial: definiciones y características esenciales}

En esta sección, se describen y discuten algunos elementos teóricos que caracterizan la RSE, en diferentes contextos. En este sentido, Chumaceiro et al, (2013:309-321), determinan que la RSE comprende dos aspectos, una interna asociada al recurso humano, evidenciado como competencia y calificación del trabajador y otra asociada a la dimensión externa que se relaciona con las comunidades locales, socios comerciales, proveedores, consumidores, problemas ecológicos y desarrollo sostenible. Por su parte, Camacho et al, (2002:313), señalan que los elementos de la RSE son: los trabajadores, consumidores, competidores, administración pública, entorno geográfico, humano y medio ambiente; como aquellos grupos que merecen la consideración de ser tenidos en cuenta. De esta manera, La RSE gana importancia en el sentido de reflejar la conciencia que adquieren las empresas, cuando reconocen que sus actividades laborales pueden generan impactos positivos o negativos sobre las sociedades y el medio ambiente.

Dávila (2008:48) y Toca (2007:50) refuerzan el concepto, asumiendo que la RSE, propone invertir en capital humano sin olvidar sus obligaciones jurídicas y el cumplimiento de sus normas, dando importancia relevante a los individuos involucrados, los cuales generan, eficiencia y eficacia en el desarrollo de los procesos empresariales. Desde este punto de vista, el papel de la RSE da un paso más de la mera acción de lograr un impacto a través de acciones benéficas, debe avanzar hacia la consecución de aportes sociales que busquen mitigar la pobreza, el analfabetismo, el respeto por la naturaleza, poniendo como principio cumplir con las expectativas de los grupos interesados, en cuanto a las necesidades plasmadas en los derechos humanos, exigencias éticas y modelos de sostenibilidad, por su parte las empresas agroindustriales del sector arrocero de Villavicencio tiene el doble compromiso social; por un lado generar empleo y por otro lado, proteger a sus trabajadores, a las comunidades aledañas y al medio ambiente.

Un concepto diferente de RSE lo propone Cortina (2005:113-127) cuando determina que este concepto no es mera filantropía, reflejada en acciones de beneficencia hacia las comunidades afectadas, la empresa debe proponer el diseño de políticas que lleven a las 
Responsabilidad social de las empresas agroindustriales del sector arrocero en Villavicencio Colombia

Obando-Bastidas, Jorge Alejandro; Franco-Montenegro, Aldemar; Bohórquez-Barbosa, Tania Margarita y Figueroa-Enciso, Stefany Michel

empresas a generar actuaciones que tengan en cuenta los intereses de todos los afectados por ella. Complementando esta propuesta, Aguilera et al, (2012:126) y Barros et al, (2015:80-87) observan a la RSE como un compromiso asumido por las empresas hacia la sociedad, que involucra el beneficio del desarrollo sostenible, propiciando de esta manera un equilibrio entre el crecimiento económico y el bienestar y los trabajadores, con una intensidad similar a la generación de valor para todos los propietarios. Asimismo, Guédez (2006:206) deja en claro que las organizaciones se mantienen a largo plazo en el mercado con niveles de ganancia aceptables, cuando asumen la estrategia de RSE como parte integrante y permanente de sus fines.

Otro punto de vista propone Correa (2007:87-102), cuando señala a la RSE en diferentes ámbitos culturales, académicos y empresariales. Roitstein (2004:5-28), mira estos aspectos como una relación de socios que logre combinar los recursos y las competencias de la empresa y del conjunto de actores sociales en pos del desarrollo de relaciones mutuamente beneficiosas por su parte De la Cuesta González, (2004:45-58) Nieto et al, (2004:28-39) y Volpentesta, (2009:280) ubican este concepto en el marco de la defensa de los recursos naturales, lo que genera costos económicos, pero propicia la formación de valores culturales, sociales, económicos, ambientalistas y jurídicos, que benefician a diferentes actores.

Desde otra mirada no menos importante, Garavito (2012: 81-104) señala que la RSE, puede ser vista como un costo o como una inversión, este sentido Romero, (2010:456-467); aporta que, en la RSE, sólo consideran los problemas económicos sin tomar en cuenta la multiplicidad de elementos que interactúan. De esta manera la (RSE) se ha convertido en parte fundamental de la cultura, el mundo académico y empresarial, ya que mejora la imagen corporativa, así lo mira Alvarado et al, (2008:37-59) cuando afirma que "las imágenes de las empresas son elaboradas por la vía de la percepción, la experiencia, las construcciones mentales y las de la memoria".

En síntesis, para el caso específico en la presente comunicación, la Responsabilidad Social parte de una actitud consciente y responsable que asumen las empresas arroceras hacia el bienestar común, compartido con los trabajadores, el medio ambiente y todos los actores sociales que hacen parte del entorno físico que rodean dichas empresas.

\section{Responsabilidad social en empresas agroindustriales del sector arrocero en Villavicencio: análisis estadístico}

Esta investigación es de tipo cuantitativo, abordada desde los métodos de la correspondencia estadística. En este método las unidades de variables que caracterizan a la responsabilidad social de las empresas frente a los trabajadores y a la comunidad aledaña, se corresponden de una manera exploratoria y significativa, con los elementos de la escala Likert. La exploración se hace mediante graficas de correspondencias, mientras que la significancia se aborda desde la relación de cada una de los elementos de las variables de estudio, con las valoraciones de la escala. Dicha relación corresponde al cumplimiento de la 
prueba Chi-cuadrado.

La encuesta se aplicó a 4 empresas del sector arrocero de la ciudad de Villavicencio. En este contexto se implementan los instrumentos a dos tipos de poblaciones: 1) Trabajadores de las empresas; 2) Población que conforma la comunidad aledaña a estas empresas. Se eligen empresas vigentes y de amplio espectro en el sector arrocero de Villavicencio. Se realizó un muestreo probabilístico estratificado, ya que la población total se dividió en dos partes (Trabajadores y comunidad aledaña). Los sujetos de estudio se seleccionaron aleatoriamente formando dos estratos proporcionales a la cantidad de trabajadores y a la cantidad de familias que viven alrededor de estas empresas. Finalmente, a cada empresa, proporcionalmente a su tamaño se le asigna una cantidad de trabajadores y de población aledaña, a los que finalmente se le aplican los instrumentos. La tabla 1, muestra la cantidad de encuestas aplicadas.

\section{Tabla 1}

Muestreo

\begin{tabular}{lcc}
\hline \multicolumn{1}{c}{ Empresa } & Trabajadores & Población aledaña \\
\hline Agrocom & 40 & 92 \\
Inproarroz & 50 & 84 \\
Procearroz S.A & 78 & 130 \\
Procesadora de Arroz Montecarlo & 70 & 156 \\
Subtotal & 238 & 462 \\
\hline \multicolumn{2}{c}{ TOTAL } & $\mathbf{7 0 0}$ \\
\hline
\end{tabular}

Fuente: Elaboración propia (2017).

Método de las correspondencias: Matemáticamente, esta técnica propone elementos de carácter geométrico, a través de gráficos sencillos, que explican la correlación, el acercamiento y la discriminación de las variables (1. Características de la Responsabilidad social de las empresas frente a los trabajadores; 2. responsabilidad social de las empresas frente a la comunidad aledaña) hacia los elementos de la escala Likert.

El método es importante porque se reconoce desde la descripción grafica el acercamiento de cada elemento de las variables hacia la valoración de la escala, estableciendo visualmente una relación y desde la inferencia la significancia en un p-valor inferior a 0.05 que garantiza la veracidad de esta relación (Obando y Castellanos: 375: 382). Mediante la correlación estadística se definen las relaciones entre las características de un fenómeno, el grado de esta relación, manifestada en el peso de los ejes sobre el cual se distribuyen las variables que 
Responsabilidad social de las empresas agroindustriales del sector arrocero en Villavicencio Colombia

Obando-Bastidas, Jorge Alejandro; Franco-Montenegro, Aldemar; Bohórquez-Barbosa, Tania Margarita y Figueroa-Enciso, Stefany Michel

se correlacionan. A mayor peso, más aceptación del método (Martínez et al, 2009).

El instrumento aplicado es la encuesta tipo escala Likert, con 16 items, que indagan en la muestra 4 aspectos: Educación, vivienda, medio ambiente, salud y recreación. Se aplicó el instrumento tanto a las personas que laboran en las empresas, como a las personas que viven alrededor de estas, denominada población aledaña.
Para el análisis de datos se utilizó el programa estadístico R. La figura 1, evidencia las primeras correspondencias de las variables, que hace relación a las encuestas aplicadas en los diferentes aspectos, a la comunidad aledaña. En forma exploratoria se hace necesario determinar por cada grafica generada en cada componente, la inercia que genera peso en la calidad de la información propuesta por el método.

\section{Figura 1 \\ Resultados de correlacion en la comunidad}
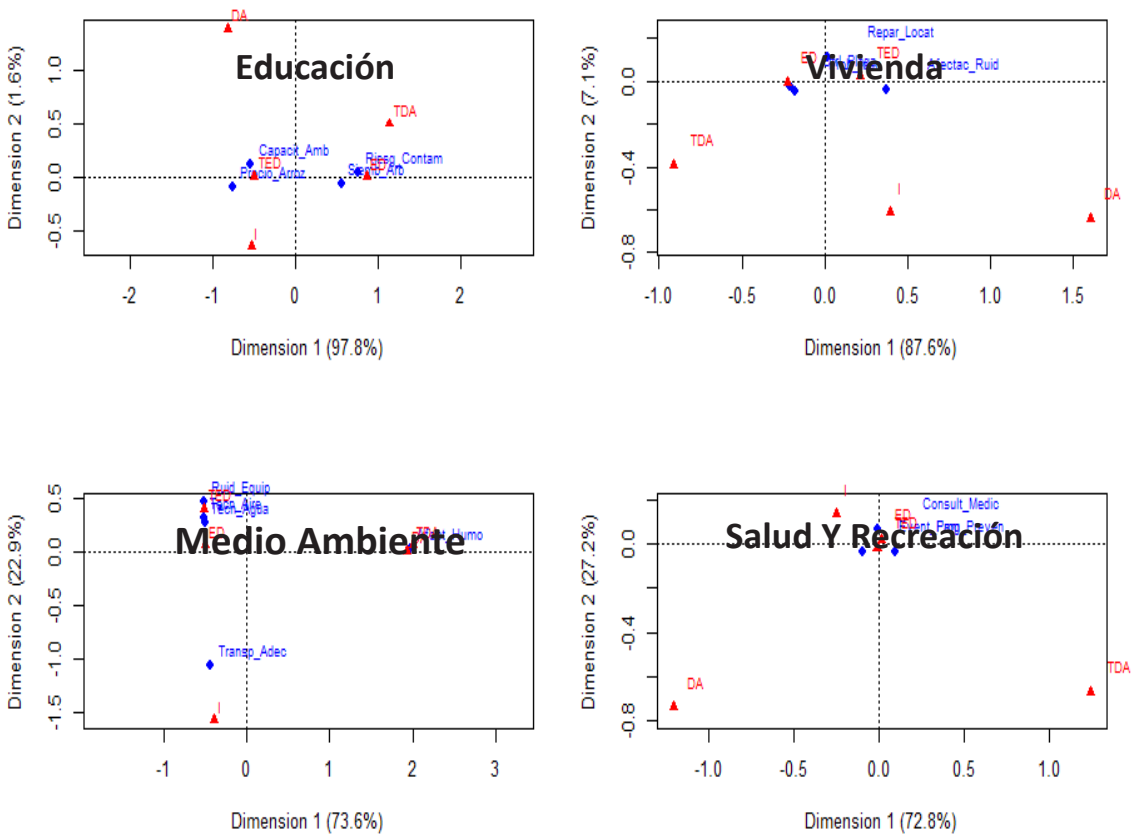

Fuente: elaboración propia 
La Figura 1, refleja la correspondencia de las variables aplicadas en la instrumentalizacion de la comunidad aledaña a las industrias arroceras de Villavicencio, mostrando una alta significancia en la informacion aportada, por el metodo. De esta manera el componente educacion esta evidenciando un $97.8 \%+1.6 \%=$ $99.4 \%$, el componente vivienda $87.6 \%$ $+7.1 \%=94.7 \%$, el componente medio ambiente $73.6 \%+22.9 \%=96.5 \%$ y el componente salud y recreacion $72.8 \%+$ $27.2 \%=100 \%$.

Con la significancia aportada, las correspondencias en la grafica permiten explorar que para el componente educacion, el $50 \%$ de las variables se asocian con la valoracion, totalmente en desacuerdo (TED), mientras que el otro $50 \%$ estan en desacuerdo (ED), exactamente la misma situacion se observa en el componente vivienda. Para los componentes de medio ambiente el $60 \%$ esta totalmente en desacuerdo y el $20 \%$ visualiza con indiferencia (I) el proceso de RSE, mientras que el $20 \%$ de la comunidad esta totalmente de acuerdo (TDA). La salud y recreacion evidencia que el $33 \%$ esta totalmente en desacuerdo mientras que el $67 \%$ esta en desacuerdo.

Para determinar la significancia de la relación de cada una de las variables, se calcula un chi-cuadrado con su respectivo p-valor que comprueba la veracidad de la relacion (tabla 2)

\section{Tabla 2}

Resultados correspondencia de la comunidad

\begin{tabular}{|c|c|c|c|c|c|c|}
\hline VARIABLES & DENOMINACIÓN & TED & ED & DA & TDA & P-Valor \\
\hline Capacit_Amb & $\begin{array}{l}\text { La empresa realiza actividades de capacitación sobre conta- } \\
\text { minación ambiental a la población circunvecina. }\end{array}$ & $\mathbf{x}$ & & & & 0,000017 \\
\hline Riesg_Contam & $\begin{array}{l}\text { La empresa mantiene informada a la comunidad sobre los } \\
\text { riesgos de la contaminación que genera. }\end{array}$ & & $\mathbf{x}$ & & & 0,000014 \\
\hline Precio_Arroz & $\begin{array}{l}\text { La empresa arrocera les vende más barato el arroz por ser } \\
\text { vecinos. }\end{array}$ & $\mathbf{x}$ & & & & 0,000011 \\
\hline Siemb_Arb & $\begin{array}{l}\text { La empresa arrocera ha realizado campañas de siembras } \\
\text { de árboles con la comunidad. }\end{array}$ & & $x$ & & & 0,00015 \\
\hline Afectac_Ruid & $\begin{array}{l}\text { La empresa le ha facilitado ayuda para que en su vivienda } \\
\text { no se vea afectado por el ruido. }\end{array}$ & $\mathbf{x}$ & & & & 0,0016 \\
\hline Repar_Locat & $\begin{array}{l}\text { La empresa ayuda en las reparaciones locativas de las vivien } \\
\text { el hollín que arroja las quemas de residuos arroceros. }\end{array}$ & as por & $\mathbf{x}$ & & & 0,00013 \\
\hline Ctrl_Plaga & $\begin{array}{l}\text { La empresa desarrolla un plan de control de plagas para el } \\
\text { beneficio de la comunidad aledaña. }\end{array}$ & $\mathbf{x}$ & & & & 0,000011 \\
\hline Prob_Inun & $\begin{array}{l}\text { La empresa arrocera les ayuda a solucionar problemas de } \\
\text { inundación a causa del taponamiento que provoca la cas- } \\
\text { carilla. }\end{array}$ & & $\mathbf{x}$ & & & 0,00000 \\
\hline Ruid_Equip & $\begin{array}{l}\text { El ruido que producen los equipos de las empresas arroce- } \\
\text { ras ha disminuido desde que usted vive aquí. }\end{array}$ & & & $\mathbf{x}$ & & 0,00003 \\
\hline
\end{tabular}


Responsabilidad social de las empresas agroindustriales del sector arrocero en Villavicencio Colombia

Obando-Bastidas, Jorge Alejandro; Franco-Montenegro, Aldemar; Bohórquez-Barbosa, Tania Margarita y Figueroa-Enciso, Stefany Michel

\section{Cont... Tabla 2}

\begin{tabular}{|c|c|c|c|c|}
\hline Tecn_Agua & $\begin{array}{l}\text { Considera usted que las empresas arroceras usan nuevas } \\
\text { tecnologías para evitar la contaminación del agua. }\end{array}$ & $x$ & & 0,00001 \\
\hline Afect_Humo & $\begin{array}{l}\text { El humo que producen los molinos arroceros afecta la co- } \\
\text { munidad de su barrio. }\end{array}$ & & $x$ & 0,00007 \\
\hline Tecn_Aire & $\begin{array}{l}\text { Considera usted que las empresas arroceras han realizado } \\
\text { mejoras tecnológicas para no contaminar el aire. }\end{array}$ & $\mathbf{x}$ & & 0,00000 \\
\hline Transp_Adec & $\begin{array}{l}\text { Los camiones que transportan el arroz son adecuados y se } \\
\text { encuentran en perfecto estado. }\end{array}$ & $x$ & & 0,002 \\
\hline Event_Fam & $\begin{array}{l}\text { La empresa patrocina u organiza eventos deportivos para } \\
\text { las familias del sector. }\end{array}$ & $x$ & & 0,00002 \\
\hline Prog_Preven & $\begin{array}{l}\text { La empresa patrocina u organiza programas de prevención } \\
\text { y promoción. }\end{array}$ & & $\mathbf{x}$ & 0,00004 \\
\hline Consult_Medic & $\begin{array}{l}\text { La empresa presta ayuda con medicamentos o consultas } \\
\text { médicas y otros a las personas que resultan afectadas por } \\
\text { la contaminación que ellos generan. }\end{array}$ & & $x$ & 0,00001 \\
\hline
\end{tabular}

\section{Fuente: Elaboración propia (2017).}

Con valores que bajo un nivel de confianza del 95\%, determinan una relación entre las variables y los valores de la escala, dadas por el P-valor, se evidencia una correspondencia validada. De esta manera el componente de la educación muestra una baja valoración que lleva a las siguientes percepciones: La empresa no realiza actividades de capacitación sobre contaminación ambiental a la población circunvecina, no informa a la comunidad sobre los riesgos de la contaminación que genera. Así mismo la comunidad no percibe que se le venda más barato el arroz por ser vecinos, tampoco realiza campañas de siembras de árboles con la comunidad.

Con respecto al componente vivienda, las correlaciones indican también una baja valoración, de esta manera para la comunidad la empresa no ayuda en las reparaciones locativas de las viviendas por el hollín que arroja las quemas de residuos arroceros, no hace control de plagas que nacen de las actividades propuestas en los molinos de arroz, ni mucho menos facilita ayuda para que en su vivienda no se vea afectado por el ruido. Asimismo, la empresa no toma medidas para el control del ruido, ni tampoco le interesan los problemas de inundación a causa del taponamiento que provoca la cascarilla.

Desde el componente que relaciona el medio ambiente no se observa en forma significativa, una valoración que favorezca a las empresas arroceras. Las correlaciones indican 
que estas empresas no disminuyen los ruidos de sus equipos, no hace uso de nuevas tecnologías que le permitan evitar la contaminación del agua y del aire, no implementan medidas para que el humo no llegue hasta las casas vecinas, aumentando la sensación de contaminación, hasta los camiones en los cuales se transporta el arroz no son adecuados y contribuyen a aumentar el panorama de contaminación ambiental.

De la misma manera, la valoración determinada por la comunidad refleja la poca participación de la empresa en la organización de eventos deportivos, programas de prevención, en ayudas médicas para problemas derivados de la contaminación generada en el procesamiento industrial del arroz.

A continuación, en la figura 2 , se muestran las correspondencias entre las características de la responsabilidad social de las empresas hacia los trabajadores y las unidades de valoración de la escala Likert.

\section{Figura 2}

\section{Resultados de correlacion en los trabajadores}
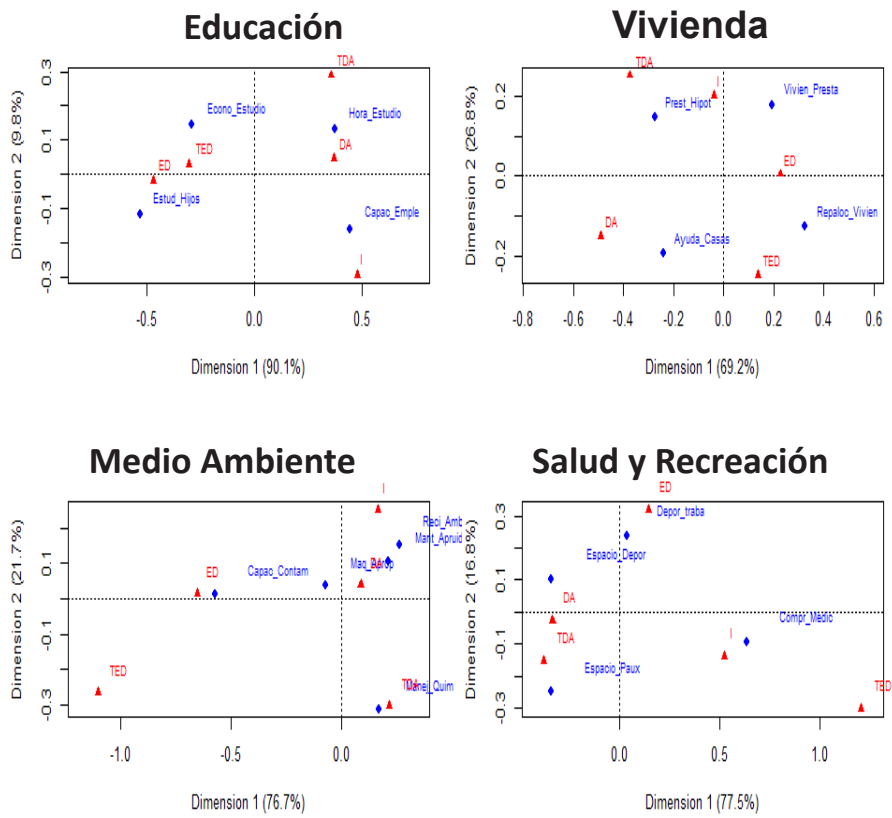

Fuente: Elaboración propia (2017). 
Responsabilidad social de las empresas agroindustriales del sector arrocero en Villavicencio Colombia

Obando-Bastidas, Jorge Alejandro; Franco-Montenegro, Aldemar; Bohórquez-Barbosa, Tania Margarita y Figueroa-Enciso, Stefany Michel

Desde la consulta generada en la aplicación de la encuesta a los trabajadores de la empresa, la tabla 3 refleja la correspondencia de las variables aplicadas en la instrumentalizacion de la trabajadores de las industrias arroceras de Villavicencio, mostrando una alta significancia en la informacion aportada por en los ejes por el metodo. De esta manera el componente educacion esta evidenciando un $90.1 \%+9.8 \%=$ $99.9 \%$, el componente vivienda $69.2 \%$ $+26.8 \%=96 \%$, el componente medio ambiente $76.7 \%+21.7 \%=98.4 \%$; y el componente salud y recreacion $77.5 \%+$ $16.8 \%=94.3 \%$.

Con la significancia aportada, las correspondencias en la grafica permiten explorar que para el componente educacion el $50 \%$ de las variables se asocian con la valoracion en desacuerdo (ED) y en totalmente en desacuerdo (TED), el $25 \%$ es indiferente (I) , mientras que el otro $25 \%$ estan de acuerdo (DA). Para el componente de Vivienda el $50 \%$ esta en desacuerdo (ED) y el $25 \%$ visualiza con indiferencia (I) y el restante $25 \%$ esta de acuerdo (DA). El proceso de RSE, el componente de medio ambiente el $40 \%$ esta de acuerdo (DA), el $20 \%$ totalmente de acuerdo (TDA), mientras que el restante $40 \%$ se muestra indiferente (I) y en desacuerdo (ED). La salud y recreacion evidencia que el $50 \%$ esta en desacuerdo acuerdo (ED) e indiferente (I), mientras que el restante $50 \%$ se muestra totalmente de acuerdo (TDA) y de acuerdo (DA).

\section{Tabla 3}

Resultados correspondencia de los trabajadores

\begin{tabular}{|c|c|c|c|c|c|c|c|}
\hline VARIABLES & DENOMINACIÓN & TED & ED & I & DA & TDA & P-Valor \\
\hline Econo_Estudio & $\begin{array}{l}\text { La empresa ayuda económicamente para realizar estu- } \\
\text { dios a todo nivel educativo }\end{array}$ & $x$ & & & & & 0,00006 \\
\hline Hora_Estudio & $\begin{array}{l}\text { La empresa facilita horarios para adelantar estudios a } \\
\text { todo nivel educativo. }\end{array}$ & & & & $x$ & & 0,00004 \\
\hline Capac_Emple & $\begin{array}{l}\text { La empresa genera capacitación a sus empleados en } \\
\text { todos los aspectos inherentes a su actividad. }\end{array}$ & & & $\mathbf{x}$ & & & 0,00011 \\
\hline Estud_Hijos & $\begin{array}{l}\text { La empresa facilita el estudio a los hijos de los emplea- } \\
\text { dos por medio de becas y subsidios. }\end{array}$ & & $x$ & & & & 0,0005 \\
\hline Vivien_Presta & $\begin{array}{l}\text { La empresa facilita la adquisición de vivienda por medio } \\
\text { de préstamos. }\end{array}$ & & & $\mathbf{x}$ & & & 0,001 \\
\hline $\begin{array}{l}\text { Repaloc_Vi- } \\
\text { vien }\end{array}$ & $\begin{array}{l}\text { La empresa ayuda en las reparaciones locativas de vi- } \\
\text { viendas de sus empleados. }\end{array}$ & & $x$ & & & & 0,0003 \\
\hline Ayuda_Casas & $\begin{array}{l}\text { La empresa facilita mediante ayudas o prestamos la } \\
\text { construcción de casas en lotes de sus trabajadores. }\end{array}$ & & & & $x$ & & 0,0001 \\
\hline Prest_Hipot & $\begin{array}{l}\text { La empresa facilita el pago de préstamos hipotecarios a } \\
\text { sus trabajadores. }\end{array}$ & & & & & $\mathbf{x}$ & 0,0008 \\
\hline $\begin{array}{l}\text { Capac_Con- } \\
\text { tam }\end{array}$ & $\begin{array}{l}\text { La empresa realiza capacitación a los agricultores para } \\
\text { evitar la contaminación de aguas. }\end{array}$ & & $\mathbf{x}$ & & & & 0,0003 \\
\hline Reci_Amb & $\begin{array}{l}\text { La empresa genera proceso de reciclaje como medida } \\
\text { de cuidado del medio ambiente. }\end{array}$ & & & $\mathbf{x}$ & & & 0,0004 \\
\hline
\end{tabular}




\section{Cont... tabla 3}

\begin{tabular}{lllc}
\hline Mant_Apruid & $\begin{array}{l}\text { La empresa hace mantenimiento a sus aparatos gene- } \\
\text { radores de ruidos. }\end{array}$ & $\mathbf{X}$ & 0,0006 \\
\hline \multirow{2}{*}{ Maq_Aprop } & $\begin{array}{l}\text { La empresa tiene la maquinaria apropiada para evitar la } \\
\text { contaminación ambiental en su procesamiento. }\end{array}$ & $\mathbf{X}$ & 0,0005 \\
\hline \multirow{2}{*}{ Manej_Quim } & $\begin{array}{l}\text { La empresa propicia el buen uso, cuidado y manejo de } \\
\text { los químicos usados en el procesamiento del arroz. }\end{array}$ & $\mathbf{X}$ & 0,003 \\
\hline Depor_traba & $\begin{array}{l}\text { La empresa patrocina eventos deportivos para los traba- } \\
\text { jadores y sus familias. }\end{array}$ & $\mathbf{X}$ & 0,0005 \\
\hline Espacio_Depor & $\begin{array}{l}\text { La empresa posee espacios físicos para promover el } \\
\text { deporte de sus trabajadores y o usuarios. }\end{array}$ & $\mathbf{X}$ \\
\hline Espacio_Paux & $\begin{array}{l}\text { La empresa posee espacios dotados con los elementos } \\
\text { necesarios para los primeros auxilios. }\end{array}$ & 0,0004 \\
\hline Compr_Medic & $\begin{array}{l}\text { La empresa contribuye en la compra de medicamentos } \\
\text { fuera del POS. }\end{array}$ & $\mathbf{X}$ & 0,0006 \\
\hline
\end{tabular}

Fuente: Elaboración propia (2017).

Con valores que bajo un nivel de confianza del 95\%, determinan una relación entre las variables y los valores de la escala, dadas por el P-valor, se evidencia una correspondencia validada para las respuestas brindadas en el proceso de encuestas a los trabajadores de las empresas arroceras del Villavicencio.

De esta manera en el componente de la educación, los encuestados manifiestan que las empresas les ayudan favoreciéndoles horarios para su crecimiento intelectual, sin embargo, no reciben ayuda económica, ni para ellos, ni para su núcleo familiar, para realizar estudios en cualquier nivel educativo. Con respecto al componente vivienda, las correlaciones indican que la empresa les facilita a sus trabajadores ayudas o préstamos hipotecarios que pueden ser usados en la construcción de casas, lo préstamos no se hacen para la reparación de sus viviendas.

Entre los trabajadores y la población encuestada en el sector arrocero de Villavicencio, se evidencia que las empresas no le dan relevancia al tema de salud y recreación de los trabajadores y de la comunidad aledaña, siendo este el puntaje más bajo. Otro factor relevante es la educación puesto que en gran parte las personas encuestadas se encuentran en desacuerdo, indicando que no les brindan ayudas educativas, ni capacitaciones al respecto.

Desde el componente que hace relación al medio ambiente los trabajadores reconocen que las empresas arroceras hacen mantenimiento a sus aparatos generadores de ruidos, adquiriendo compromisos que conlleva a evitar la contaminación ambiental, haciendo un buen manejo de químicos usados en el procesamiento del arroz; sin embargo, reflejan la ausencia de capacitación a los agricultores para evitar la contaminación de aguas y de procedimientos para generar conductas de reciclaje. 
Responsabilidad social de las empresas agroindustriales del sector arrocero en Villavicencio Colombia

Obando-Bastidas, Jorge Alejandro; Franco-Montenegro, Aldemar; Bohórquez-Barbosa, Tania Margarita y Figueroa-Enciso, Stefany Michel

De la misma manera, la valoración determinada por los trabajadores, refleja la poca participación de la empresa en la organización de eventos deportivos, pero, habilita espacios físicos para la práctica de deportes, además de propiciar elementos de primeros auxilios que prevén accidentes de trabajo. El pago de hipotecas, las ayudas para la construcción se constituyen en elementos que apoyan el grado de responsabilidad emitidos por estas empresas.

\section{Conclusiones}

Para el presente trabajo se propuso evaluar el grado de responsabilidad de las empresas arroceras de Villavicencio hacia las comunidades y hacia sus trabajadores. Los resultados expresados bajo el método de las correspondencias, descritos en forma gráfica e inferencial, determinaron el valor del uso del método de correspondencias, ya que se logró examinar las diferentes correlaciones y su respectivo acercamiento hacia la valoración de los elementos de las unidades de variable hacia la escala Likert. El p-valor del chicuadrado, dio cuenta de la alta relación de las valoraciones emitidas por los diferentes actores intervinientes en este proceso de investigación.

Los resultados encontrados evidencian un paralelo, en donde la comunidad responde con proposiciones que hacen ver el poco grado de responsabilidad social en los aspectos relacionados con la educación, la vivienda, la salud y el escaso compromiso de estas empresas con el medio ambiente. Desde el punto de vista de los trabajadores existe un compromiso leve en los procesos de educación, es bueno que estos tengan horarios apropiados para que asistan a colegios y universidades. El problema se presenta cuando no se observan el mismo compromiso con sus familias.

Desde el punto de vista de los trabajadores las empresas generan también principios de RSE, cuando existe una valoración significativa a los procesos de mantenimiento a sus aparatos generadores de ruidos y el uso de maquinaria apropiada para evitar la contaminación ambiental en su procesamiento. Ante la protección del medio ambiente la comunidad evidencia serias dificultades, sobre todo porque en la región en donde se desarrolla la investigación las inundaciones en los barrios en épocas de lluvias son frecuentes y las empresas no limpian los accesos por donde corren los residuos de la cascarilla del arroz. Aspectos como el ruido, la descontaminación del agua, el humo frecuente de estos molinos, son elementos que a la concepción de los encuestados las empresas salen poco favorecidas.

Generalizando, en todos los aspectos desde la comunidad, los procesos de responsabilidad social (educación, vivienda, medio ambiente, salud y recreación) por parte de estas empresas presentan serias dificultades, no se nota el compromiso de estas para la comunidad, desde el punto de vista de los trabajadores se observan indicios en donde la presencia de la empresa hace notar armonía con sus trabajadores.

\section{Bibliografía citada}

Aguilera, Adriana, y Puerto, Doria (2012), Crecimiento empresarial basado en la Responsabilidad Social. Pensamiento y Gestión. No. 32. Barranquilla, Colombia Pp. 1-26.

Alvarado, Alejandro, y Schlesinger, Ma- 
ría. (2008), Dimensionalidad de la responsabilidad social empresarial percibida y sus efectos sobre la imagen y la reputación: una aproximación desde el modelo de Carroll. Estudios Gerenciales, Vol. 24, No. 108. Cali, Colombia. Pp. 37-59.

Barros, Milena; Retamozo, Melissa, y González, David (2015), Responsabilidad Social Empresarial. Semilla Dice. No 1. Barranquilla, Colombia. Pp. 80-87.

Camacho, Idelfonso; Fernández, José; González, Raúl y Miralles Josep (2002), Ética de la empresa. Bilbao, España. Desclée De Brouwer. Pp. 313.

Chumaceiro, Ana; Hernández, Judith; Yori, Ligia; y Ziritt, Gertrudis (2013), Responsabilidad Social Empresarial y Políticas Públicas. Revista de Ciencias Sociales. Vol. XIX, No. 2. Maracaibo, Venezuela. Pp. 309-321.

Correa, Juan (2007), Evolución histórica de los conceptos de responsabilidad social empresarial y balance social. Semestre Económico. Vol. 10, No. 20. Medellín, Colombia. Pp. 87-102.

Cortina, Adela (2005), Ética de la empresa, no sólo responsabilidad social. Revista Portuguesa de Filosofía. Vol. 20. Portugal. Pp. 113-127.

Dávila, Aracely., y Gómez, Jose (2008), Responsabilidad social empresarial: un reto para la industria química. Synthesis. Vol. 1. Chihuahua, México. Pp. 48

De la Cuesta González, Marta. (2004), El porqué de la responsabilidad social corporativa. Boletín económico de ICE. Vol. 2813. España. Pp. 45-58.

FEDEARROZ. (2010), Arroz. Revista Arroz.Vol. 58. No. 484. Colombia. Pp.9

Garavito, Cecilia. (2012), Responsabi- lidad social empresarial y mercado de trabajo. Economía. Perú. Vol. 31, No. 61. Pp. 81-104.

Guédez, Víctor. (2006), Ética y Práctica de la Responsabilidad Social Empresarial: El aporte de la empresa al Capital Social. Caracas, Venezuela. Editorial Planeta Venezolana. Pp. 206.

Hernández, Hector; Obando, Jorge; y Montoya, Juan (2016), Las innovaciones técnicas y administrativas como dimensiones de la innovación organizacional. In Vestigium Ire,10(2), Pp 133-146.

Martínez, Ross; Tuya, Leonel; Martínez, Mercedes; Pérez, Alberto; y Cánovas, Ana. (2009), El coeficiente de correlación de los rangos de Spearman caracterización. Revista Habanera de Ciencias Médicas, 8(2), 0-0.

Namakforoosh, Mohammad. (2005), Metodología de la investigación. México. Limusa. Pp. 525.

Nieto, Mariano; y Fernández, Roberto (2004), Responsabilidad social corporativa: la última innovación en management. Universia Business Review. No. 1. Madrid, España. Pp. 28-39.

Obando, Jorge; y Castellanos, María (2014), Análisis de correspondencia. El caso de la evaluación por competencias. Primer encuentro colombiano de educación estocástica. La enseñanza y el aprendizaje de la probabilidad. En línea: http://funes.uniandes.edu.co/6558/1/Obando2014An\%C3\%A1lisisECEE.pdf Consultado, 21-05-2017.

Roitstein, Florencia (2004), La responsabilidad social empresarial en Argentina: tendencias y oportunidades. Academia Revista Latinoamericana de Administración. No. 32. Bo- 
Responsabilidad social de las empresas agroindustriales del sector arrocero en Villavicencio Colombia

Obando-Bastidas, Jorge Alejandro; Franco-Montenegro, Aldemar; Bohórquez-Barbosa, Tania Margarita y Figueroa-Enciso, Stefany Michel

gotá, Colombia. Pp. 5-28.

Romero, Nestor (2010), La responsabilidad social corporativa: ¿una estrategia de cambio hacia un modelo ético de desarrollo? Revista de Ciencias Sociales. Vol. XVI, No. 3. Maracaibo, Venezuela. Pp. 456-467.
Toca, Claudia (2007), Desarrollos Teóricos para la Comprensión y el Análisis de Stakeholders. Bogotá, Colombia. Documentos de administración. Pp. 50.

Volpentesta, Jorge (2009), Gestión de la responsabilidad social empresaria Argentina. Intermilenio. Pp. 280.

- $\quad$ Esta obra está bajo una licencia de Creative Commons Reconocimiento-NoComercial- Compartirlgual 3.0 Unported. http://creativecommons.org/licenses/by-nc-sa/3.0/deed.es_ES 